\title{
Kaposi's varicelliform eruption in herpes simplex encephalitis
}

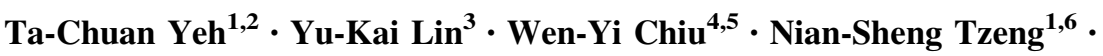 \\ Te-Yu Lin ${ }^{2,7}$
}

Received: 9 October 2015/Accepted: 12 November 2015/Published online: 25 November 2015 (C) SIMI 2015

\begin{abstract}
A 28-year-old man with a history of atopic dermatitis developed a widespread, erythematous rash on his middle abdomen, bilateral thigh, and chest for 1 week. He had complained of a severe headache, and gradually became drowsy, and therefore he had been sent to the emergency department (ED). In the ED, the patient presented with a fever of $39^{\circ} \mathrm{C}$, a tonic-clonic seizure, disorganized behavior and a reduction in consciousness with a Glasgow Coma Scale score of 8 . Physical examination revealed generalized, erosive, monomorphic vesicles as well as a coalescing skin rash, except for the palms, soles, and the genital region (Fig. 1a). The patient had a head computed tomography (CT scan) after his condition was stabilized,
\end{abstract}

Wen-Yi Chiu

hanrogyi@gmail.com

1 Department of Psychiatry, Tri-Service General Hospital, School of Medicine, National Defense Medical Center, Taipei, Taiwan, ROC

2 Department of General Medicine, Tri-Service General Hospital, School of Medicine, National Defense Medical Center, Taipei, Taiwan, ROC

3 Department of Neurology, Tri-Service General Hospital, National Defense Medical Center, Taipei, Taiwan, ROC

4 Department of Family Medicine, Zouying Branch of Kaohsiung Armed Forces General Hospital, Kaohsiung, Taiwan, ROC

5 Department of Internal Medicine, Kaohsiung Armed Forces General Hospital, Kaohsiung, Taiwan, ROC

6 Student Counseling Center, National Defense Medical Center, Taipei, Taiwan, ROC

7 Division of Infectious Diseases and Tropical Medicine, Department of Internal Medicine, Tri-Service General Hospital, School of Medicine, National Defense Medical Center, Taipei, Taiwan, ROC but there were no significant findings of the initial scan. Under the impression of a CNS infection, immediate lumbar puncture was performed in the ED to get an early diagnosis. Cerebrospinal fluid (CSF) analysis showed a predominance of lymphocytes, elevation of red blood cell (lymphocyte count $8 / \mu \mathrm{L}, \mathrm{RBC}$ count $352 / \mu \mathrm{L}$ and total cell counts $360 / \mu \mathrm{L}$ ), there was a normal opening pressure, and there was no traumatic tap during the procedure. The CSF glucose level was $80 \mathrm{mg} / \mathrm{dL}$ (serum glucose level was $101 \mathrm{mg} / \mathrm{dL}, \mathrm{CSF} / \mathrm{blood}$ ratio $>0.6$ ), and the CSF total protein was $27 \mathrm{mg} / \mathrm{dL}$. A complete blood count analysis revealed a leukocytosis (WBC count $18.68 \times 10^{3} / \mu \mathrm{L}$ ); an elevation of C-reactive protein $(6.37 \mathrm{mg} / \mathrm{L})$ was also observed. A T2 fluid-attenuated inversion recovery (FLAIR) magnetic resonance imaging of the brain showed asymmetric hyperintensity over both temporal lobes (Fig. 1b). The imaging studies supported the diagnosis of herpes simplex encephalitis (HSE), and intravenous acyclovir was started immediately. Laboratory examinations confirmed the diagnosis subsequently after admission.

Herpes simplex encephalitis (HSE) is the most common cause of sporadic viral encephalitis that is characterized by a severe headache, fever, seizures, and a decline in consciousness. In adults, the majority of cases are caused by HSV-1, which is believed to represent the reactivation of latent HSV in situ within the central nervous system. The diagnosis of herpes simplex encephalitis is based on clinical features (a triad of fever, headache and an altered mental state), cerebrospinal fluid examination, and brain imaging [1]. HSV DNA detection by polymerase chain reaction using the cerebrospinal fluid has a sensitivity of $98 \%$ and a specificity of 94-100\%. Edematous changes in the temporal lobe and high red cell count in the CSF are typical features of HSE, and were observed in our patient [2]. The association between poor outcome and the 
Fig. 1 a Dome-shaped, crusted, erythematous and umbilicated vesicles over the abdomen with an underlying atopic dermatitis. b Hyperintensity corresponding to asymmetric edematous changes of the cortical and subcortical region of bilateral medial temporal lobes, insula, and subfrontal area. $\mathbf{c}$ T2 FLAIR magnetic resonance imaging of a normal brain
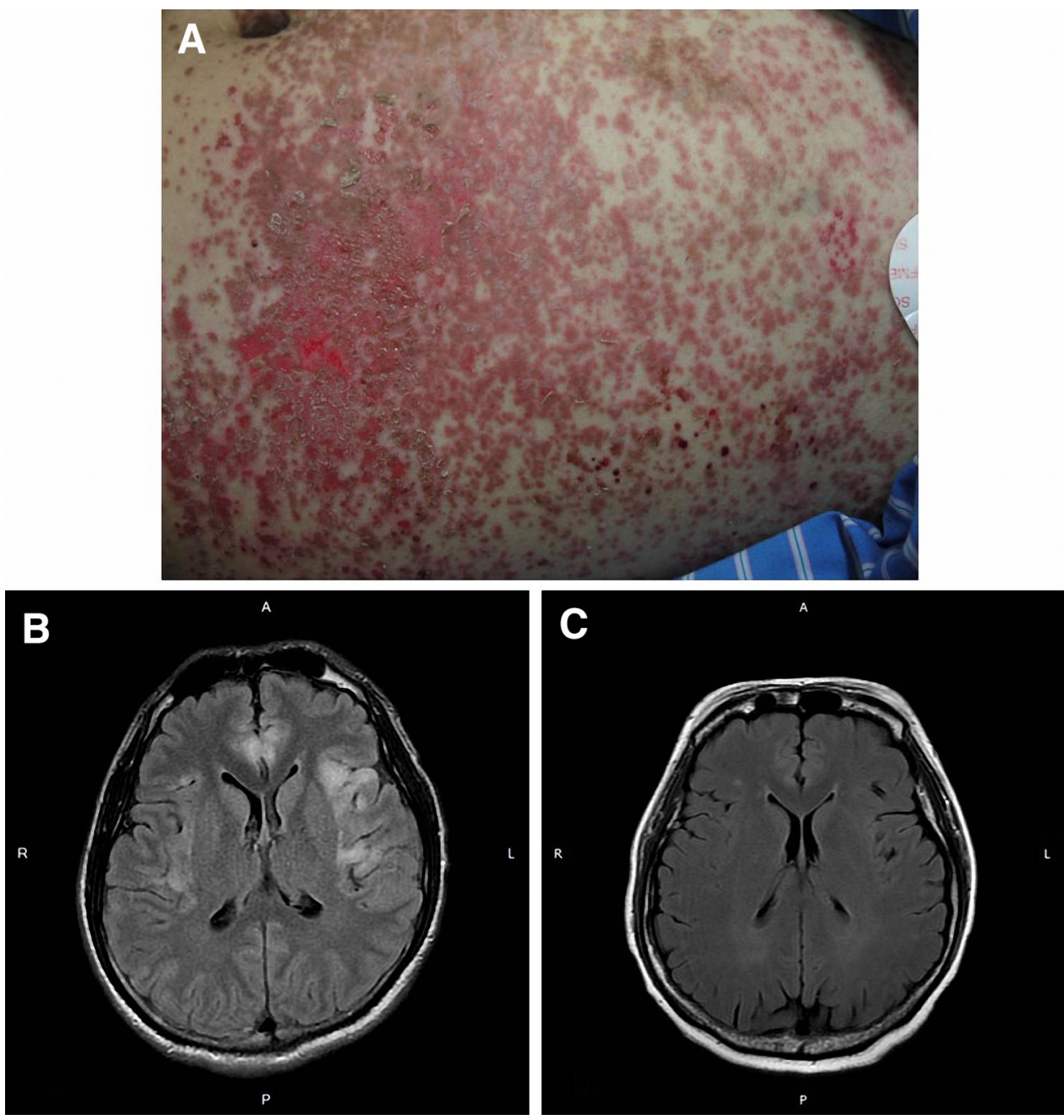

presence of red blood cells in CSF has been reported, which may be result from a severe immunological reaction [2]. The mortality rate occasionally exceeds $70 \%$ without antiviral medication. Prompt initiation of therapy with acyclovir within $72 \mathrm{~h}$ is important to obtain maximal clinical benefit, and to reduce the mortality rate to $<30 \%$ [3].

The neuropsychiatric symptoms of HSE are associated with anatomic structures, such as the fronto-orbital region, hippocampus, cingulate gyrus and insular cortex. The temporal and orbitofrontal lobes involvement may also elicit personality changes, anosmia, or olfactory or gustatory hallucinations in the acute phase [4]. The inflammatory process of the limbic cortex may also manifest in disorganized behavior and emotional dysregulation occurring during the course of HSE. An altered mental state is often preceded by a period of delirium [4]. We did not observe the other mentioned psychotic symptoms except for disorganized behavior in our patient, which might be related to his level of consciousness during the initial evaluation.
Kaposi's varicelliform eruption (KVE), also known as eczema herpeticum, is the dissemination of HSV in the setting of pre-existing inflamed skin, typically involving atopic dermatitis. KVE is often misdiagnosed as an exacerbation of the patient's pre-existing skin lesion. Its diagnosis is generally established by Tzanck testing. Once KVE is suspected, it is imperative to initiate antiviral treatment because of high mortality rate of the untreated encephalopathy [5].

In conclusion, we present the case of a man with the combination of herpes simplex encephalitis and Kaposi's varicelliform eruption, which may mimic a skin rash caused by a bacterial infection in the acute care setting. This unusual presentation indicates a severe infection and a poor prognosis. An immediate lumbar puncture is essential and helpful for establishing the correct diagnosis, and initiating helpful therapy.

\section{Compliance with ethical standards}

Conflict of interest The authors declare that they have no conflict of interest. 
Statement of human and animal rights The authors hereby declare that the research documented in the present manuscript, involving human participant, has been carried out in accordance with the ethical standards of the institutional and national research committee and have been performed in accordance with the ethical standards laid down in the 1964 Declaration of Helsinki and its later amendment or comparable ethical standards.

Informed consent Written informed consent was obtained from the patient.

\section{References}

1. Studahl M, Lindquist L, Eriksson BM et al (2013) Acute viral infections of the central nervous system in immunocompetent adults: diagnosis and management. Drugs 73(2):131-158
2. Poissy J, Champenois K, Dewilde A et al (2012) Impact of Herpes simplex virus load and red blood cells in cerebrospinal fluid upon herpes simplex meningo-encephalitis outcome. BMC Infect Dis $12: 356$

3. Solomon T, Michael BD, Smith PE et al (2012) Management of suspected viral encephalitis in adults-Association of British Neurologists and British Infection Association National Guidelines. J Infect 64(4):347-373

4. Wiedlocha M, Marcinowicz P, Stanczykiewicz B (2015) Psychiatric aspects of herpes simplex encephalitis, tick-borne encephalitis and herpes zoster encephalitis among immunocompetent patients. Adv Clin Exp Med 24(2):361-371

5. Studdiford JS, Valko GP, Belin LJ et al (2011) Eczema herpeticum: making the diagnosis in the emergency department. J Emerg Med 40(2):167-169 